\title{
Size-dependent tradeoffs in aggressive behavior towards kin
}

$9 \quad \dagger$ corresponding authors

11 22chloe.a.fouilloux@jyu.fi; ORCID 0000-0003-1265-5824

12 3lutz.fromhage@jyu.fi; ORCID 0000-0001-5560-6673

13 4janne.k.valkonen@jyu.fi; ORCID 0000-0002-2177-6612

14 5bibiana.rojas@jyu.fi; ORCID 0000-0002-6715-7294

17 Keywords: kin discrimination; cannibalism; tadpole; poison frog; larval aggression

18 Short title: Tradeoffs in larval aggression

Abstract.

21 Aggression between juveniles can be unexpected, as their primary motivation is to survive until their

22 reproductive stage. However, instances of aggression, which may escalate to cannibalism, can be vital

23 for survival, although the factors (e.g. genetic or environmental) leading to cannibalism vary across 
taxa. While cannibalism can greatly accelerate individual growth, it may also reduce inclusive fitness

when kin are consumed. As a solution to this problem, some cannibals demonstrate kin discrimination

and preferentially attack unrelated individuals. Here, we used both experimental and modeling

approaches to consider how physical traits (e.g. size in relation to opponent) and genetic relatedness

mediate aggressive behavior in dyads of cannibalistic Dendrobates tinctorius tadpoles. We paired

sibling, half-sibling, and non-sibling tadpoles of different sizes together in an arena and recorded their

aggression and activity. We found that the interaction between size and relatedness predicts aggressive

31 behavior: large non-siblings are significantly more aggressive than large siblings. Unexpectedly,

although siblings tended to attack less overall, in size mismatched pairs they attacked faster than in

non-sibling treatments. Ultimately, it appears that larval aggression reflects a balance between

relatedness and size where individuals trade-off their own fitness with that of their relatives.

\section{Introduction}

37 Cannibalism has often been understood to be a response to stressful environmental conditions (e.g.

low-food, high competition) that is modulated by physical (e.g. size, condition) and genetic variables.

39 It was not until the late-twentieth century that the behavior was critically evaluated and one-off observations and anecdotes (Jenkins and Carpenter, 1946; Merritt Hawkes, 1920) were synthesized

41 into a review about the trends and occurrence of cannibalism (Fox, 1975). More recent investigations

42 of cannibalism have recorded and tested its manifestation across a wide variety of clades, 43 environmental states, and life history stages (Van Allen et al., 2017; Barkae et al., 2014; Van den 44 Beuken et al., 2019; Cooper et al., 2015; Schulte and Mayer, 2017). Aggression, a behavior often 45 tested as the precursor to cannibalistic behavior, can occur in resource-abundant, low-density 46 environments (Fox, 1975; Mock et al., 1987; Summers and Symula, 2001), and the effect of commonly 
47 tested variables (i.e. starvation, competition) on eliciting cannibalistic behavior does not follow a

48 consistent trend on either class-wide or even family-specific levels. Ultimately, for a behavior

49 expressed in almost every clade in the animal kingdom, it remains difficult to tease apart the

50 evolutionary motivation behind cannibalism.

Aggression in cannibals can be difficult to interpret, as the motivation is not always hunger-driven, and death is not always the end-state of aggressive interactions. For example, cannibalistic lizards

(Podarcis gaigeae) in Greece show local variation in aggression, where males are more aggressive in

high-density (but high-resource) islands. It appears that aggression by males leads to the consumption

of conspecific tails, which although is not always deadly, serves as a high-fat meal that decreases the

sexual quality of male competitors (Cooper et al., 2015). Aggression between juveniles complicates

matters further, as individuals are not competing for mates and usually do not hold territories. For the

most part, juvenile aggression and cannibalism are justified by competition for immediate nutritional

resources (larval flounders: Dou et al., 2000; earwig nymphs: Dobler and Kölliker, 2011), or parental

care (vulture chicks: Margalida et al., 2004) which may be survival mechanisms to compensate for

fluctuating environmental conditions (Mock et al., 1987).

63
Theoretical justifications predicting cannibalism are often built around physical qualities. Across the animal kingdom, many studies have found that cannibals are most often larger than their prey (Barkae et al., 2014; Claessen et al., 2004; Ibáñez and Keyl, 2010; Rojas, 2014) although exceptions exist when larger individuals are weakened (Richardson et al., 2010). Kinship between individuals has also been used to characterize cannibalistic interactions. This has been shown to be an important factor in several cannibalistic species who demonstrate kin discrimination and avoid eating kin (salamanders: Pfennig et al., 1994, bulb mites: Van den Beuken et al., 2019, toad tadpoles: Pfennig and Frankino, 1997), 
71 though this is far from a rule, as similar range of cannibals do consume their kin without avoidance

72 (moths: Boots, 2000, poison frog tadpoles: Gray et al., 2009). Despite kinship and physical

73 attributes driving much of the discussion on the evolution and maintenance of cannibalism, few

74 studies have incorporated both in predicting cannibalism outside of oophagy or sexually competitive

75 contexts. Moreover, such studies have so far been limited to invertebrates: in European earwigs,

76 kinship and weight asymmetry affected cannibalistic behaviour both separately and in an interaction,

77 such that weight asymmetry effects were stronger among unrelated individuals (Dobler and Kölliker,

78 2011). In both desert (Bilde and Lubin, 2001) and wolf spiders (Roberts et al., 2003), kinship but not

79 weight asymmetry affected cannibalism. While fascinating in their own right, these studies hardly

80 provide a basis for generalising to distant clades such as vertebrates.

81 Dendrobates tinctorius is a species of Neotropical poison frog whose larvae are aggressive cannibals

82 (Rojas, 2014). Tadpoles are often deposited by their fathers in ephemeral pools of water, where they

83 are left to develop until metamorphosis (Rojas and Pašukonis, 2019). Although tadpoles are most often

84 transported singly, the ephemeral pools in which they are deposited can have multiple tadpoles of

85 various developmental stages (Rojas and Pašukonis, 2019) and degrees of relatedness (B. Rojas \& E.

86 Ringler, unpublished data). In these environments, cannibalism is common (Rojas, 2014, 2015), yet

87 the escalation of aggression to cannibalism in this species has not been explicitly tested. Closely related

88 poison frogs have linked aggressive behavior and cannibalism (Gray et al., 2009; Summers and

89 Symula, 2001), though exceptions exist in obligate egg-feeders with parental care (Dugas et al., 2016).

90 For D. tinctorius, the costs of aggression are direct, as consuming kin reduces inclusive fitness and the

91 potential for injury (even with a small counterpart) is high; the potential benefits, on the other hand,

92 are complex, as by shortening time to metamorphosis and increasing physical size thereafter,

93 individuals may be able to escape precarious conditions and improve fitness prospects. 
94 In this study we tested physical and genetic variables to better understand the basis of aggression in a

95 cannibalistic species. We conducted behavioral assays between pairs of Dendrobates tinctorius

96 tadpoles, and measured aggression and activity in response to size differences and relatedness. These

97 tests will allow us to evaluate the importance of physical characteristics with respect to genetic

98 relatedness as predictors of aggression. Experiments were supplemented with theoretical models based

99 on inclusive fitness theory to study the predictors of aggression in this species; together, these

100 experiments and models contribute to our understanding of how cannibalism is shaped by the costs

101 and benefits of relatedness and aggression in animals.

\section{Methods}

Dendrobates tinctorius is a species of Neotropical poison frog with elaborate parental care. Males and Pašukonis, 2019). Tadpoles of this species are omnivorous and frequently demonstrate cannibalistic behaviour (Rojas, 2014, 2015); despite this, it is not unusual to see tadpoles of various

111 stages coexisting within the same pool in the wild (Rojas and Pašukonis, 2019).

113 We used tadpoles from a breeding laboratory population of $D$. tinctorius kept at the University of

114 Jyväskylä, Finland. We maintained a paternal half-sibling design as it could be expected that paternal

115 half-siblings are more likely to co-occur as a result of fathers reusing pools after multiple transport

116 events. Tadpole dyads were assigned in response to (1) individuals needing to be visually

117 distinguishable from each other (i.e. size), and (2) the laboratory mating schedule/network, which was 
118 prioritized as to not stress the animals from overbreeding. Adult pairs were each housed in a 55L

119 terrarium that contained layered expanded clay, leaf-litter, moss substrate and were equipped with a

120 shelter, logs, and live plants. Terraria were maintained at $26 \mathrm{C}( \pm 2 \mathrm{C})$ and were automatically misted

121 with reverse osmosis water four times a day (maintaining a humidity around 95\%) and lit with a 12:12

122 photoperiod. Frogs were fed live Drosophila fruit flies coated in vitamin supplements five times per

123 week. Tadpoles were raised singly in $10 \times 6.5 \times 5 \mathrm{~cm}$ containers which were filled with spring water,

124 and fed ad libitum a diet of fish food (JBL NovoVert flakes) three times a week. Adult and tadpole

125 health and water levels were checked daily.

126

127

128

129

\section{Behavioral trials}

Pairs of tadpoles of different degrees of relatedness (full sibling, half-sibling, non-sibling) were placed together in an arena. Tadpoles in early larval development were used, but we established a cut-off point before the toe differentiation in hind legs development to control for possible life-history effects (stage 31; Gosner, 1960). Experimental tadpole weight ranged from $0.04 \mathrm{~g}$ to $0.38 \mathrm{~g}$, and size differences between pairs ranged from $0.03 \mathrm{~g}$ to $0.30 \mathrm{~g}$. Blinding in the experiment was not possible, as the set-up and experiment were conducted by the same person, but the order of trials was assigned randomly. The arena was a $18.5 \mathrm{~cm}$ by $12 \mathrm{~cm}$ clear plastic container filled with $400 \mathrm{~mL}$ of spring water; four quadrants were delineated on the base of the arena to provide information about tadpole activity throughout the experiment. Initially, each tadpole was placed on either side of an opaque partition dividing the arena; this partition kept tadpoles separated but allowed water to flow throughout the container. After an acclimation period of one hour, tadpole activity (resting, swimming) of the separated individuals was recorded every 15 seconds for 10 minutes. 
141 Following the acclimation and separated observation, the barrier was removed and tadpole interactions

142 were recorded for 60 minutes. Focal behaviors (resting, swimming, biting, and chasing; see Table 1

143 for descriptions) were recorded for both tadpoles every 15 seconds. Tadpoles were visually 144 distinguishable from each other as a result of size differences. Individuals were photographed and 145 weighed before the beginning of each trial to establish initial tadpole condition, and were only used 146 once $\left(\mathrm{n}_{\text {Trial }}=15\right.$ for each relatedness level, $\mathrm{n}=90$ tadpoles for the entire experiment).

148 Trials were ended prematurely if tadpoles demonstrated aggression levels that would cause severe 149 damage or death (where bites lasted for more than 2 seconds, recorded as "potential lethal attack"). 150 Although aggression was common, potential lethal attacks were rare, occurring in only 3/45 trials. 151 There were no tadpole deaths as a result of the behavioral trials, and all tadpoles were kept and reared 152 in the laboratory after the experiment. Assays were done according to the Association for the Study of 153 Animal Behaviour's guidelines for the treatment of animals in behavioural research and teaching 154 (ASAB 2017), and with the approval of the National Animal Experiment Board at the Regional State 155 Administrative Agency for Southern Finland (ESAVI/9114/04.10.07/2014).

\begin{tabular}{|l|l|}
\hline Behavior & Description \\
\hline Resting & Non-movement under normal, healthy conditions \\
\hline Swimming & $\begin{array}{l}\text { Movement of the tadpole's tail in over two consecutive oscillations without 'intent' } \\
\text { (see below) with respect to the other tadpole. Sometimes tadpoles contract their } \\
\text { qualify as swimming in this experiment. }\end{array}$ \\
\hline
\end{tabular}




\begin{tabular}{|l|l|}
\hline Biting & $\begin{array}{l}\text { A tadpole attempting or successfully grasping/clutching any body part of another. This } \\
\text { behavior was allowed if it was isolated and its duration lasted less than } 2 \text { seconds. If } \\
\text { the bite was followed by a "hold" by a tadpole that could result in permanent injury or } \\
\text { death of the counterpart, the trial was terminated and categorized as ended by a means } \\
\text { of "potential lethal attack". }\end{array}$ \\
\hline Chasing & $\begin{array}{l}\text { A tadpole swimming after its counterpart with intent; "intent" was perceived by the } \\
\text { observer as rapid changes in swimming speed and direction of swimming being } \\
\text { towards the other tadpole }\end{array}$ \\
\hline
\end{tabular}

Table 1. Categorization of behaviour observed during aggression trials between tadpoles.

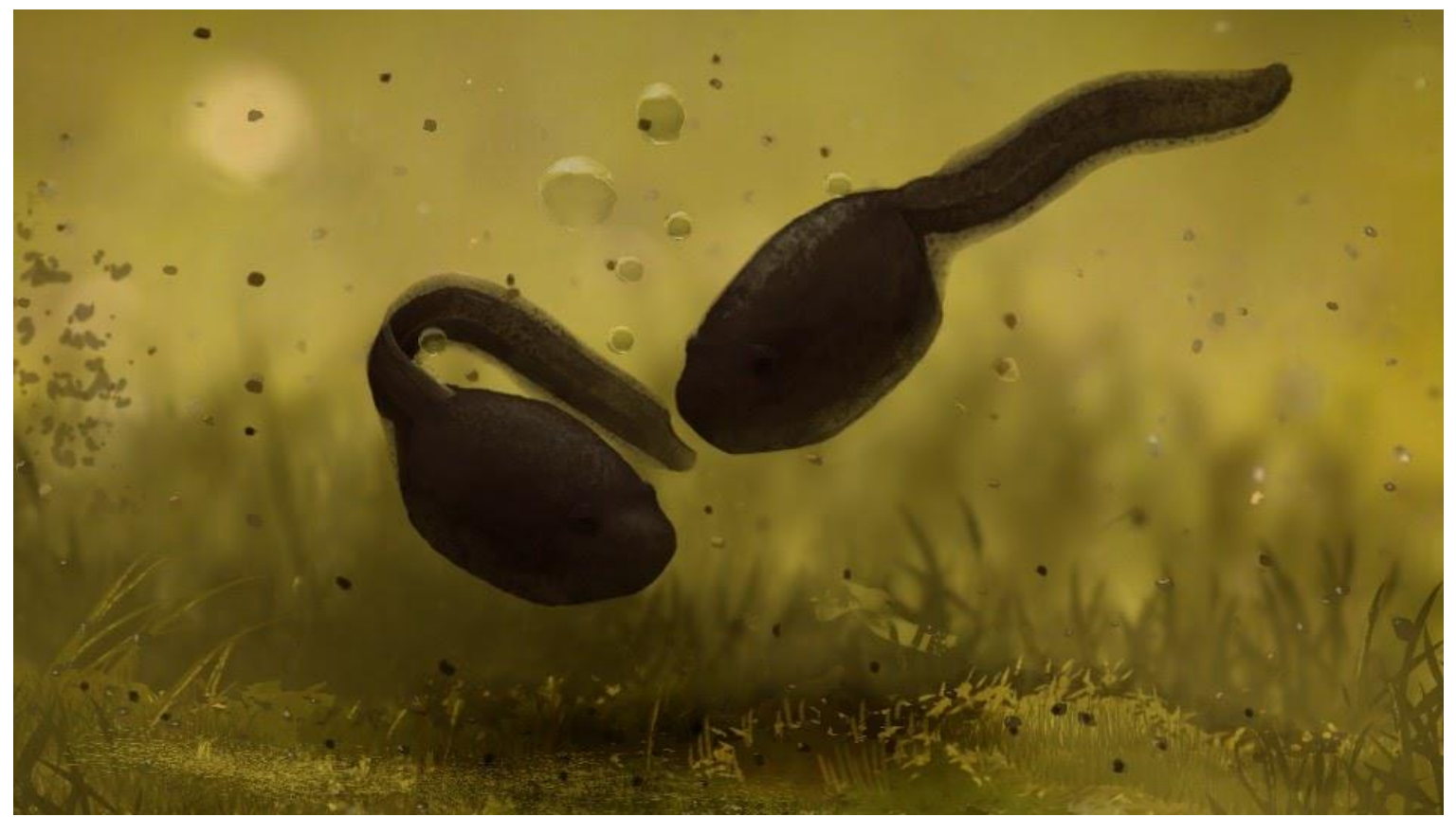

159 Image 1. Illustration of aggressive exchange between D. tinctorius tadpoles. Note the tail bite on one 160 of the tadpoles, which is a common occurrence both in wild and laboratory encounters. Published 161 with artist permission. 


\section{Statistical analysis}

164 All models and statistics were performed in the program R (v. 3.6.1, R Development Core Team,

165 2019) with additional packages "glmmTMB” (Magnusson et al., 2020), “coxme” (Therneau, 2020), 166 “dplyr” (Wickham et al., 2018), “tidyr” (Wickham et al., 2019). Activity and aggression analyses (see 167 below) took into account pair identity (Pair_ID) and family (Breeding pair) level random effects 168 (Supp. tables 1 and 2 for AIC comparisons). Differences in duration of trials during experiments $(n=$ $1693 / 45$ trials ended early due to potential lethal attacks) were taken into account by offsetting models 170 with a trial duration. Aggression and activity models were chosen based on the Akaike Information 171 Criterion (AIC, Akaike, 1973), which compares fit based on log-likelihood and the number of model 172 parameters (see supplementary materials for model AIC comparisons). We considered both negative 173 binomial (with linear and quadratic parameterization) and poisson distributions as model families in 174 AIC comparisons. Residual diagnostics and overdispersion were checked and corrected using the 175 "DHARMa" (Hartig, 2020) package.

176 Activity levels

177 Tadpole activity was categorized as "resting" and "swimming" (see Table 1 for details). Tadpole 178 activity was observed during post-acclimation (10 minutes) and experimental (max. 60 minutes) 179 periods. Activity was coded as counts and were modeled in a generalized linear mixed model 180 framework (GLMM). The best fitting model used a negative binomial linear parameterization where 181 activity was predicted by relative tadpole size (a categorical variable (i.e. small, large), where size is 182 relative within pairs) and relatedness (a categorical variable with three levels: full sib, half sib, non 183 sib) (see Supplementary Table 1). 
Aggression between tadpoles was observed as chasing or biting (see Table 1), which were recorded as counts. These two behaviors were combined to represent "total aggression". The best fitting model for aggression was a generalized linear mixed effect model (GLMM) with a negative binomial linear parameterization. Total aggression was predicted by relatedness (a categorical variable with three levels: full sib, half sib, non sib) and size (a categorical variable (i.e. small, large), where size is relative within pairs)(see Supplementary Table 2), pairs were taken into account as a random variable. We had modeled mass difference (a continuous predictor) between tadpoles as potential predictors which resulted in less parsimonious models (see Supplementary Table 2).

\section{Latency to first bite}

195 We modeled latency to first biting behavior using a mixed effect Cox proportional hazards model.

196 Survival object was parameterized with respect to latency to first bite event and absolute biting (0/1, 197 where 0 represents no biting occurred during the trial) in response to the interaction of relatedness and 198 mass difference between tadpole dyads. Latency data was built by selecting the "first biter" within a 199 pair which involved subsetting the original data set. As a result of this, we used mass difference 200 between large and small tadpoles (instead of categorical size) to incorporate trials without aggressive 201 behavior. Mass difference was calculated as the difference between the large and small tadpoles within 202 pairs: this value was always positive since large tadpoles were always more massive. Using subsetted 203 data, each pair identity was independent, so only "Family" was used as a random variable.

\section{Game theory model}

206 We modeled pairwise interactions between tadpoles arbitrarily labelled as 1 and 2 . We assumed that 207 only one tadpole per pair survives ('wins'), and that the probability of winning depends on each 208 individual's competitive strength. Competitive strength $\theta_{i}$ of tadpole $i$ was calculated based on its size, 
$s_{i}$ and its aggressiveness, $a_{i}$ as $\theta_{i}=s_{i} \cdot a_{i}$. This multiplicative formulation reflects the biological idea that a given increment in aggressiveness should have a greater effect on a large than a small tadpole's

211 competitive strength. Individual 1's probability of winning is given by its relative competitive

strength, as $\omega_{1}=\frac{\theta_{1}}{\left(\theta_{1}+\theta_{2}\right)}$. The reproductive success ("direct fitness", $v_{i}$ ) of the winning tadpole was

213 modeled under three alternative assumptions: $(1 a) v_{i}$ is size-independent, as $v_{i}=1-a_{i}{ }^{2} ;(1 b) v_{i}$ is

214 proportional to size (for a given level of aggressiveness), as $v_{i}=s_{i}-a_{i}{ }^{2}$; and (lc) $v_{i}$ is size-

215 dependent due to aggressiveness being costlier for smaller tadpoles, as $v_{i}=1-\left(\frac{a_{i}}{s_{i}}\right)^{2}$ (see Fig 3 for

216 visualization). In all three formulations costs increased at an accelerating rate, such that low levels of

217 aggression had low costs whereas high levels of aggression could be extremely costly; this was done

218 to account for the increasing danger and energy expense associated with more violent behaviors.

219 Finally, the inclusive fitness of the surviving tadpole was calculated as $v_{1}-r v_{2}$, where $r$ is the 220 relatedness between the pair. This formulation reflects the idea that winning involves the killing of a 221 relative who would have had reproductive success $v_{2}$ had it survived. The inclusive fitness of the losing 222 tadpole is zero, because the losing tadpole neither reproduces nor affects the other tadpole's 223 reproduction. We calculated the expected (i.e., probability-weighted mean) inclusive fitness of tadpole 2241 as $F_{1}=\omega_{1}\left(v_{1}-r v_{2}\right)$. For given values of $s_{1}, s_{2}$, and $a_{2}$ we numerically determined individual 1 's 225 optimal aggression level as the value of $a_{1}$ that maximises its expected inclusive fitness. By computing 226 individual 1's 'best response' aggression level for any given $a_{2}$ which its opponent might exhibit, we 227 then identified pairwise optimal aggression levels that are best responses to each other.

\section{Results}


230 We observed tadpole activity during both post-acclimation and experimental phases. While tadpoles

231 were separated by an opaque barrier during the post-acclimation phase (but water still freely moved

232 throughout the arena) we found that half-siblings demonstrated significantly less swimming behavior

233 than non-siblings (negative binomial GLMM, CI: 0.06- 1.82, $z=-2.08, p=0.037$ ) but not siblings

234 (negative binomial GLMM, CI: -0.15- 1.65, $z=1.64, p=0.102$ ). During the experiment, however,

235 we found that large tadpoles across all relatedness treatments were significantly more active than small

236 tadpoles (negative binomial GLMM, CI: $1.79-3.58, z=5.23, p<0.001$; see Fig 1, Table 1).

A

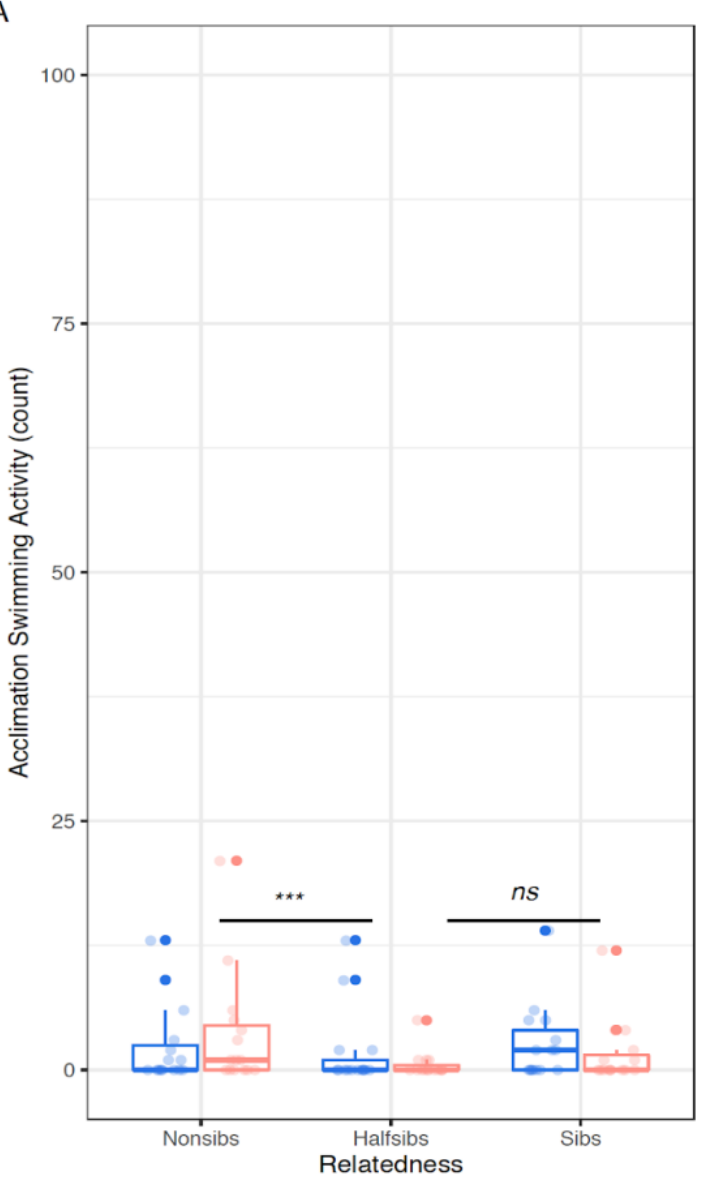

B

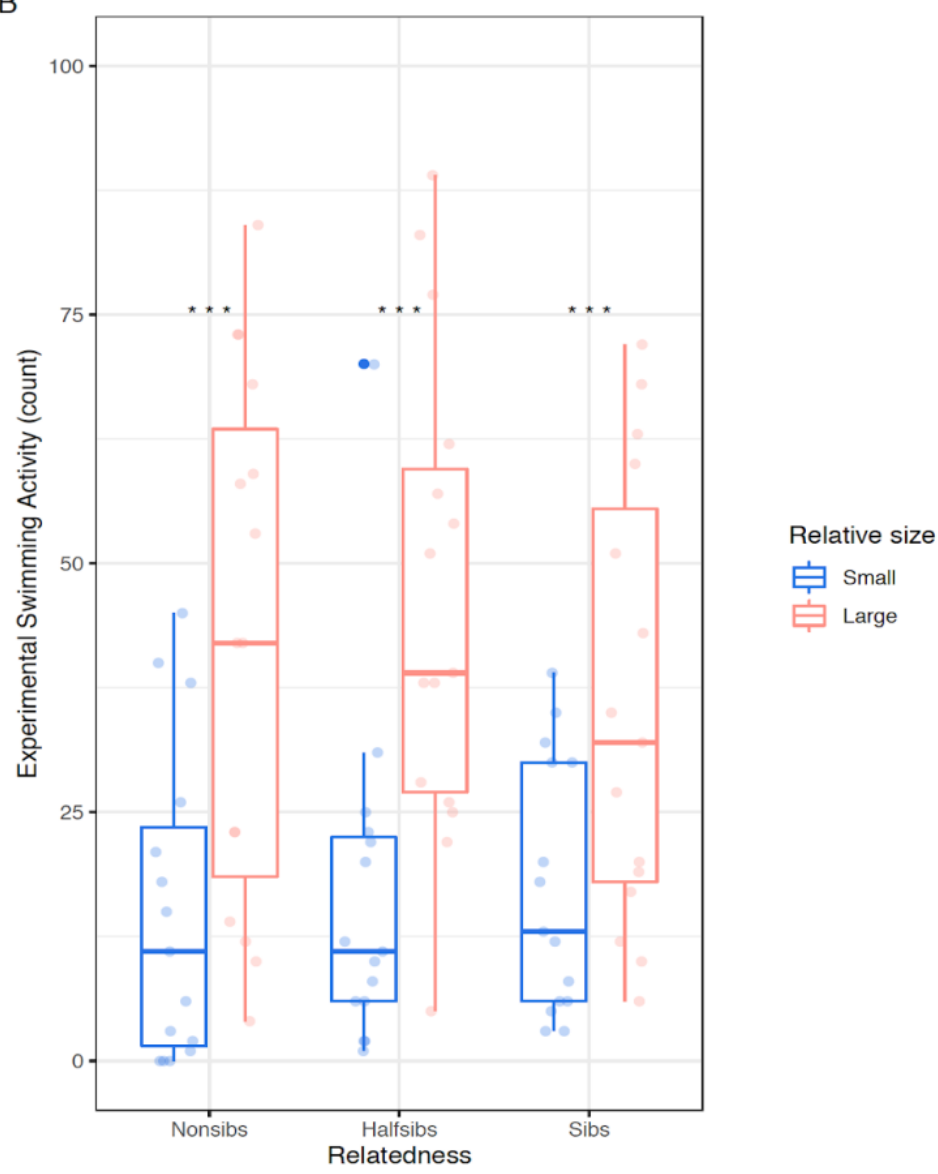

Figure 1. Tadpole activity levels before and during experimental trials. Panel (A) shows the postacclimation activity of tadpoles. We found no difference in swimming activity between large and small tadpoles during this phase, but found that half siblings were significantly less active during this period.

241 Panel $(\boldsymbol{B})$ shows experimental activity throughout behavioral trials. Large tadpoles were significantly 
242 more active than small tadpoles during assays. NTrial $=15$ for each relatedness level. Large tadpoles

243 are in pink and small tadpoles in blue. Boxplot medians are depicted by thicker lines, whiskers span

$244 \pm 1.5 *$ interquartile range.

A.

Post-acclimation Activity

B.

\section{Experimental Activity}

\begin{tabular}{|c|c|c|c|c|c|c|c|c|c|}
\hline Predictors & Estimate & $C I$ & $z$ & $p$ & Predictors & Estimate & $C I$ & $z$ & $p$ \\
\hline (Intercept) & 0.99 & $0.30-1.67$ & 2.82 & 0.005 & (Intercept) & 0.00 & $0.00-0.01$ & -29.09 & $<0.001$ \\
\hline Halfsiblings & -0.94 & $-1.82--0.06$ & -2.08 & 0.037 & Halfsiblings & 1.08 & $0.76-1.53$ & 0.43 & 0.665 \\
\hline Siblings & -0.19 & $-0.94-0.55$ & -0.50 & 0.616 & Siblings & 0.97 & $0.68-1.39$ & -0.16 & 0.874 \\
\hline Size (Large) & -0.07 & $-0.69-0.55$ & -0.22 & 0.824 & Size (Large) & 2.53 & $1.79-3.58$ & 5.23 & $<0.001$ \\
\hline \multicolumn{5}{|c|}{ Random Effects } & \multicolumn{5}{|c|}{ Random Effects } \\
\hline$\sigma^{2}$ & \multicolumn{4}{|l|}{1.71} & \multicolumn{5}{|l|}{$\sigma^{2}$} \\
\hline$\tau_{00 \text { Pair_ID }}$ & \multicolumn{4}{|l|}{0.14} & $\tau_{00 \text { Pair_ID }}$ & \multicolumn{4}{|c|}{$8.43 \times 10^{-9}$} \\
\hline$\tau_{00 \text { Family }}$ & \multicolumn{4}{|c|}{$1.82 \times 10^{-8}$} & $\tau_{00 \text { Family }}$ & \multicolumn{4}{|c|}{$5.76 \times 10^{-3}$} \\
\hline
\end{tabular}

Table 1. Summary of negative binomial GLMM with linear parameterization of tadpole activity chosen from AIC model comparison. Where (A) half-siblings demonstrated significantly less activity compared to siblings and non-siblings while tadpoles were separated during post-acclimation, which changes during the experiment $(\boldsymbol{B})$ where large tadpoles are overall more active. Models for $(A)$ and (B) were predicted by additive effects of size and relatedness. Tadpole dyads (Pair_ID) and family were accounted for as random effects, CI represents $95 \%$ confidence interval. Differences in trial time during the experiment $(n=3 / 45)$ were accounted for by using duration as offset in the model. Experimental activity model was overdispersed and size was corrected for, hence missing residual variance $\left(\sigma^{2}\right)$ in Panel $B$; $\tau$ oo represents random intercept variance.

When comparing models we found that random effects of pair ID had higher between subject variance $\left(\tau_{00}=0.14\right)$ than tadpole family $\left(\tau_{00}=1.82 \times 10^{-8}\right)$ during post-acclimation activity (Table 1 , Panel A), indicating that when separated, there was less variation in behavior on a family level. Yet, while interacting during the experiment this difference disappears (Table 1, Panel B). In both cases, 
259 between-subject variance is low, indicating that across families and pairs of tadpoles, activity levels

260 are similar.

261 Overall aggression

262 We found that large siblings were significantly less aggressive than the large non-sibling treatment,

263 exhibiting almost half the amount of aggressive behaviors as large non-siblings (Fig. 2, negative

264 binomial GLMM, $z=-2.07, p=0.039$, Table 2). Half-siblings were not significantly different from

265 either treatment. Following our expectations of creating unique pair interactions, the random effect of

266 pair identity had a high between group variation $\left(\tau_{00 \text { Pair_ID }}=0.55\right.$, Table 2$)$, but families were

267 unexpectedly consistent $\left(\tau_{00 \text { Family }}=0.06\right.$, Table 2$)$. 
bioRxiv preprint doi: https://doi.org/10.1101/2020.10.26.350132; this version posted October 26, 2020. The copyright holder for this preprint (which was not certified by peer review) is the author/funder, who has granted bioRxiv a license to display the preprint in perpetuity. It is made available under aCC-BY-NC-ND 4.0 International license.

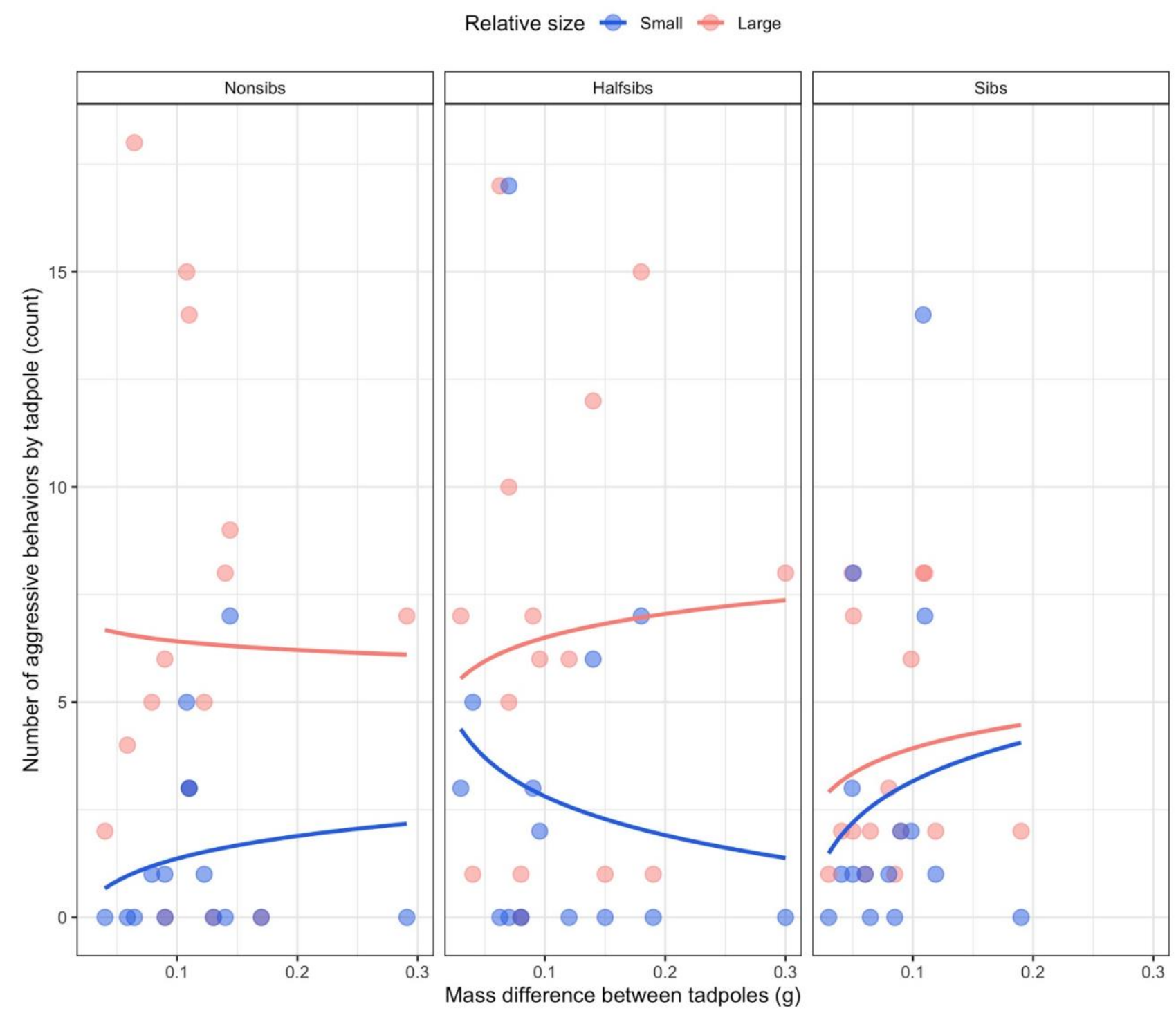

269 Figure 2. Differences in aggression across relatedness treatments with respect to weight difference 270 between dyads. Pink dots represent large tadpoles and blue dots represent small tadpoles (sizes 271 relative to dyads); NTrial $=15$ for each relatedness level. There was a higher level of aggression by 272 large tadpoles overall, but significantly less aggression by large siblings when compared to non273 siblings. Lines fit with GLM smoother $(y \sim \log (x))$ for relative sizes. 


\begin{tabular}{lcccc}
\hline \hline & \multicolumn{5}{c}{ Total aggression } \\
Predictors & Estimate & $C I$ & $z$ & $p$ \\
\hline (Intercept) & -7.95 & $-8.75--7.16$ & -19.63 & $<\mathbf{0 . 0 0 1}$ \\
Halfsiblings & 0.41 & $-0.62-1.44$ & 0.77 & 0.440 \\
Siblings & 0.58 & $-0.40-1.56$ & 1.15 & 0.249 \\
Size (Large) & 1.44 & $0.68-2.20$ & 3.71 & $<\mathbf{0 . 0 0 1}$ \\
Halfsiblings: Size (Large) & -0.44 & $-1.47-0.58$ & -0.84 & 0.399 \\
Siblings: Size (Large) & -1.02 & $-1.99--0.05$ & -2.07 & $\mathbf{0 . 0 3 9}$ \\
Random Effects & & & & \\
$\sigma^{2}$ & 0.58 & & & \\
$\tau_{00}$ Pair_ID & 0.55 & & & \\
$\tau_{00 \text { Family }}$ & 0.06 & & &
\end{tabular}

276 Table 2. Summary of negative binomial GLMM with linear parameterization of tadpole aggression.

277 Where total aggression (total count of biting and chasing) was predicted by the interaction between 278 size (two level categorical variable) and relatedness. Tadpole dyads (Pair_ID) and family were 279 accounted for as random effects, CI represents 95\% confidence interval. Differences in trial time 280 during the experiment $(n=3 / 45)$ were accounted for by using duration as offset in the model. $\sigma^{2}$ 281 represents residual variance and $\tau 00$ represents random intercept variance.

282 Latency to first bite

283 We observed that initial aggression between tadpoles can change based on physical and genetic 284 attributes. We used biting behavior as a measurement of first aggression because it consistently 285 represented the first aggressive contact in tadpole dyads. Based on a mixed effect Cox proportional 286 hazards model, we assessed the risk of first attack when considering relatedness and size difference 287 between pairs. We found a significant interaction between relatedness and size, where a higher degree 288 of relatedness led to a shorter latency to aggression when the size difference within dyads was larger. 
289 In other words, siblings demonstrated more immediate aggressive behavior towards each other when

290 their size differences were greater (Cox mixed effects, $z=2.209, p=0.022$, see Table 3). For example,

291 at a large mass difference ( $>0.15 \mathrm{~g}$ between tadpoles) siblings were more than 40 percent more likely

292 to bite than non-siblings within the first five minutes of a trial. Interestingly, non-siblings demonstrated

293 a seemingly inverted behavioral trend, where dyads with large size differences had delayed aggressive

294 behaviors. Half-siblings did not behave significantly differently from either treatment. In trials where

295 biting was exhibited, large tadpoles were most often the first aggressor $(n=8 / 13$ for siblings; $n=10 / 13$

296 for half siblings; $\mathrm{n}=11 / 12$ for non siblings).

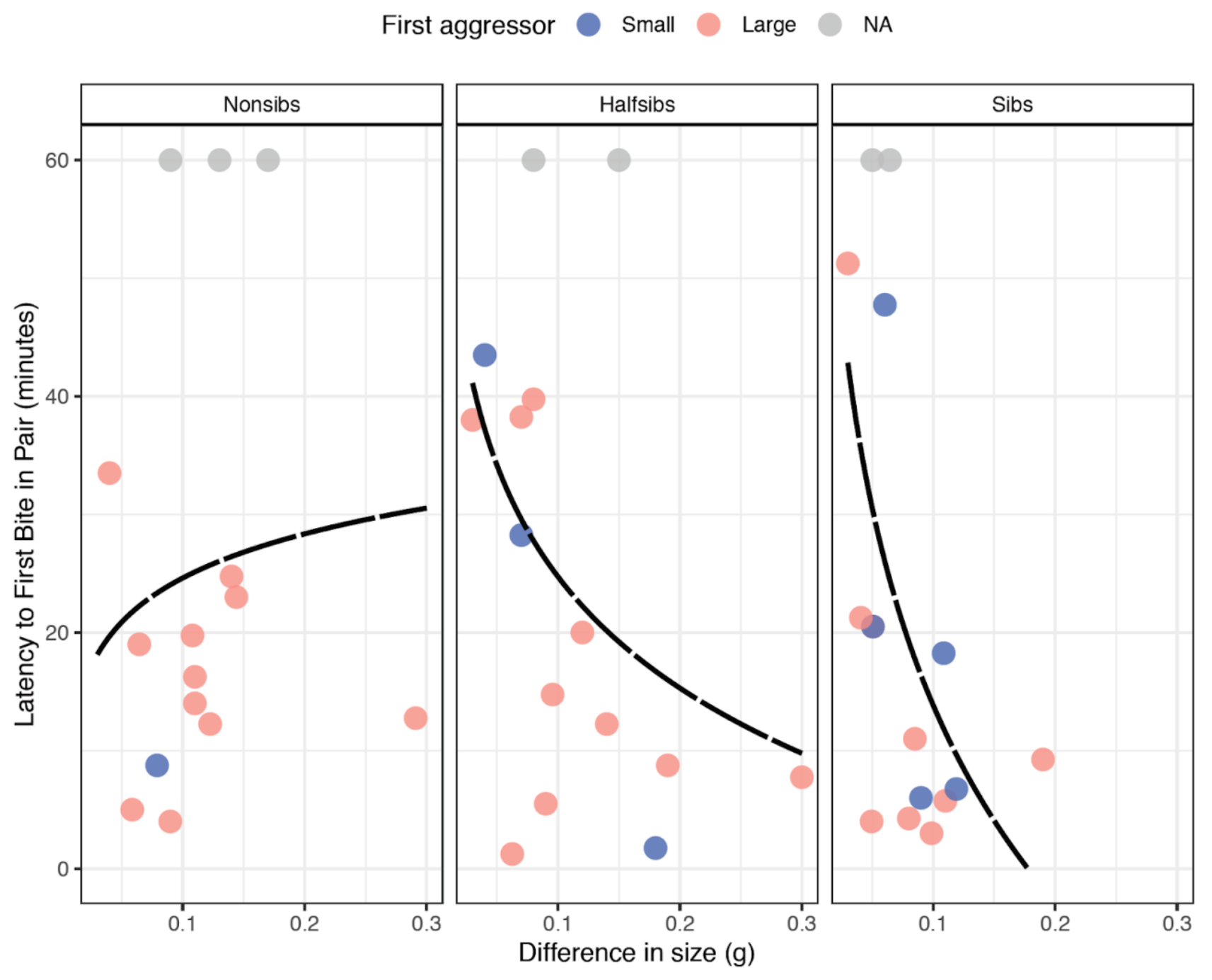


Figure 3. Latency to first bite between tadpole dyads. Points are colored by relative tadpole size within

299 dyads. Lines are fit with a GLM smoother with a $y \sim \log (x)$ formula. There is an inversion in behavior

300 as size difference increases, where sibling pairs with large size differences attacked significantly faster

301 than non-siblings. Dyads where there were no aggressive behaviors were accounted for by assigning

302 them the maximum time limit (60 minutes). $N_{\text {Trial }}=15$ for each relatedness level.

\begin{tabular}{lcccc}
\hline \hline & \multicolumn{4}{c}{ Latency to first bite } \\
Predictors & Estimate & $C I$ & $z$ & $p$ \\
\hline Halfsiblings & -1.27 & $-2.83-0.30$ & -1.59 & 0.113 \\
Siblings & -1.44 & $-3.12-0.24$ & -1.68 & 0.093 \\
Mass_Diff & 0.89 & $-8.65-10.44$ & 0.18 & 0.854 \\
Halfsiblings: Mass_Diff & 9.62 & $-2.14-21.38$ & 1.60 & 0.109 \\
Siblings: Mass_Diff & 16.32 & $1.80-30.84$ & 2.20 & $\mathbf{0 . 0 2 8}$
\end{tabular}

Table 3. Mixed effects Cox proportional hazards model. Time to first aggressive behavior was predicted by the interaction of the mass difference between tadpoles and their relatedness; family is taken into account as a random effect. There is a significant interaction between relatedness and size, where more similarly sized siblings have a shorter latency to aggression than non-siblings. Mass_Diff is the difference in weight between large and small tadpoles.

Game theory model

Based on our three formulations (1a-c) we varied the impact of size to model aggression levels of tadpoles with different degrees of relatedness. The version where aggression was both size-dependent and costlier for the smaller tadpoles (Fig 4, third row) appeared most consistent with our empirical data (Fig 2), in that larger tadpoles were consistently predicted to be more aggressive than their smaller counterparts, and overall aggression decreased with relatedness. 

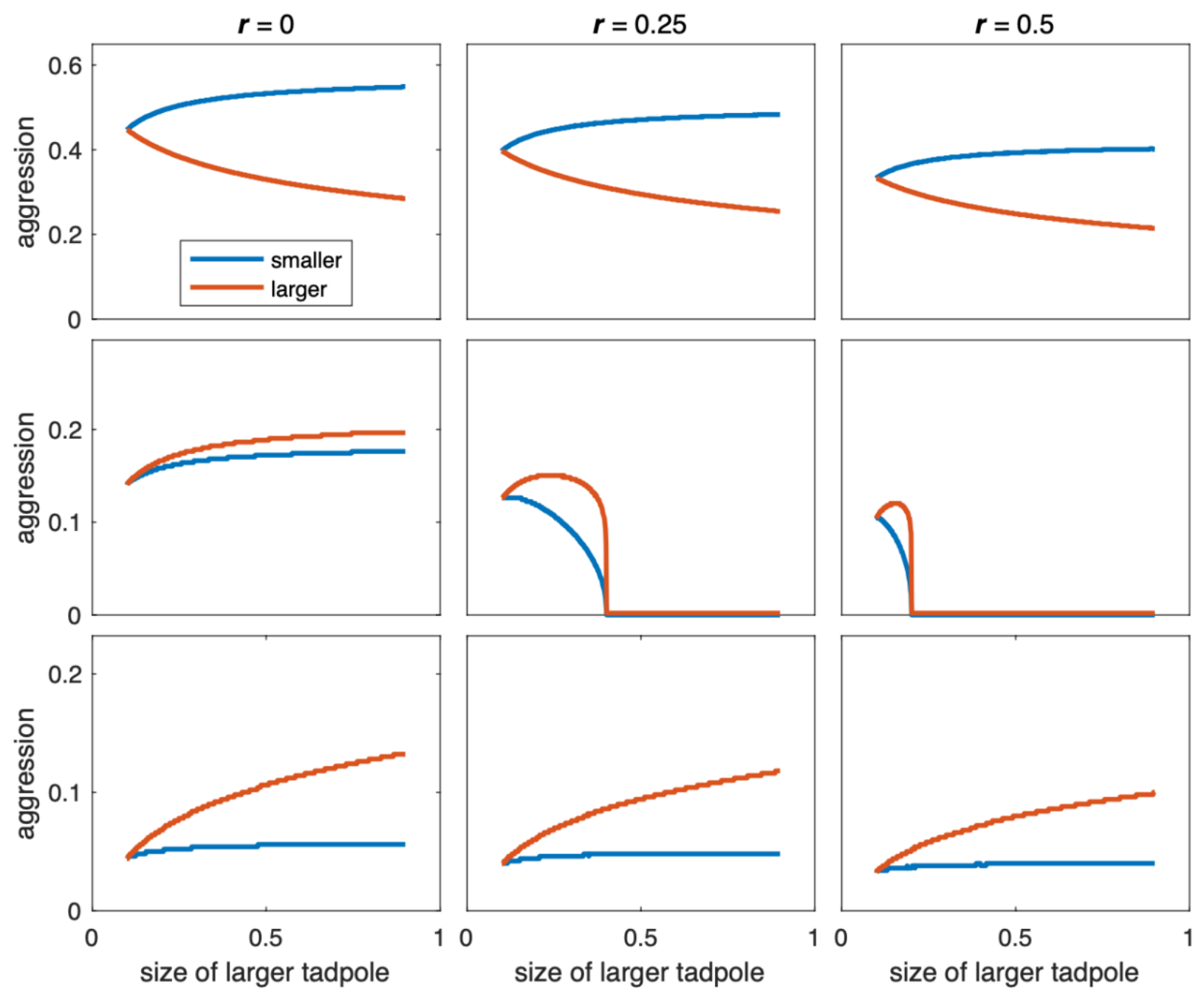

318 Figure 4. Optimal aggressiveness of dyads of tadpoles as a function of size difference for three

319 different levels of relatedness (represented in panel columns) and three sets of assumptions

320 (represented in panel rows). First row: direct fitness was assumed to be size-independent. Second

321 row: direct fitness was assumed to be proportional to size. Third row: aggressiveness was assumed

322 to be costlier for smaller tadpoles. The smaller tadpole's size was held fixed at $s_{i}=0.1$; the larger 323 tadpole's size is shown on the x-axis. 
Larval cannibalism or, more broadly, juvenile aggression has most often been studied in the context

327 of resource competition (Baras et al., 2000; Tershy et al., 2000), as juveniles do not usually defend

328 territories (Stamps and Krishnan, 1994), nor compete for sexual partners. Here, we observed

329 aggressive behaviors between $D$. tinctorius tadpoles in resource-abundant, low-density conditions. We

330 found that aggression is common (also see Fischer et al., 2020; Rojas, 2014), and depends on the

331 interaction between relative size and relatedness between tadpoles. Our results show that, together,

332 relatedness and physical attributes shape overall aggression, latency to aggression, and even activity

333 levels between dyads.

\section{Cannibalism and the environment}

336 Aggressive attacks between pairs were recorded across all relatedness treatments and sizes. Although

337 less common, small tadpoles were sometimes quicker to exhibit aggressive behaviors than their larger

338 counterparts (Fig 3) and, in some instances, were even more aggressive than large tadpoles (this was

339 observed only in sibling and half-sibling treatments, see Supplementary Fig 1). These intense 340 aggressive responses were elicited under vastly different conditions from which cannibalism is usually

341 reported, such as in response to starvation (spiders: Mayntz and Toft, 2006; cephalopods: Ibáñez and

342 Keyl, 2010; earwigs: Dobler and Kölliker, 2011), pathogens (caterpillars: Wang and Daane, 2014;

343 salamanders: Pfennig et al., 1991), high population densities (crabs: Moksnes, 2004), and in the

344 context of sexual selection (chimpanzees: Takahata, 1985; seals: Bishop et al., 2016). These

345 associations suggest that cannibalism is triggered by immediate stress and competition. However, a

346 handful of studies have been invoked in decoupling stress from aggression and cannibalism (poison

347 frogs: Dugas et al., 2016b; egrets: Mock et al., 1987, vultures: Margalida et al., 2004, snails: Baur, 
1987), contributing to the mounting evidence that across diverse taxa, immediate physical and

environmental stress are not the only triggers for cannibalistic behavior (Fox, 1975).

We report here that aggression in $D$. tinctorius is not primarily driven by immediate nutritional need.

This raises the question of whether there is a long-term fitness advantage to a cannibal that pays off as an outcome of their behavior during the larval stage (Crump, 1990; Polis, 1981).

Interactive predictors of cannibalism

Aggression between tadpoles was predicted by the interaction between relative size and relatedness within dyads. We found that large non-siblings were the most aggressive, expressing almost twice the amount of aggressive behaviors than large siblings (Fig. 2). When able to physically interact in the arena, the behavior of half-siblings did not differ significantly from the two other relatedness treatments, which demonstrates that even if there does appear to be some kin discrimination in $D$. tinctorius, it may not function on as fine of a scale as for other species (e.g., Pfennig et al., 1994).

With respect to the interaction with size, Rojas (2014) had already established that cannibalism between $D$. tinctorius tadpoles occurs faster with increasingly size-mismatched pairs. In fact, across the animal kingdom the aggressor in a pair/group is most often the larger individual (Ibáñez and Keyl, 2010; Mayntz and Toft, 2006; Mock et al., 1987). However, our findings highlight the fact that in this system aggression is not solely mediated by size differences. This suggests that there may be many other cases of context-specific discrimination across taxa that have been overlooked by not considering the interaction between relatedness and physical attributes (such as illness, injury, or phenotype). The 
context-specific kin discrimination observed in this study could have potentially evolved because the benefit to cannibals only outweighs the cost of harming kin in particular circumstances. shown to be attacked less than nonrelatives (Pfennig et al., 1994). Costs may be lower for D. tinctorius tadpoles if they do not face similar consequences of cannibalism such as acquiring pathogens (although the effects of pathogens on cannibalism in this species remain unexplored). Costs may also be decreased by the impact of parental care, whose protective effects have been shown in other poison

381 frog species (Schulte et al., 2011; but see Rojas, 2014, 2015) for seemingly counterintuitive deposition 382 choices in D. tinctorius). Although discrimination is less precise than in salamanders (Pfennig et al., 383 1994), our results support the presence of kin discrimination in D. tinctorius, and warrant further investigation into the possible proximate mechanisms regulating kin recognition in this species.

\section{Latency to aggressive behavior}

387 Latency to attack changed unexpectedly as a function of both size difference and relatedness between tadpoles. When pairs were more similarly sized, non-siblings attacked faster; in contrast, when mismatched in size, non-siblings delayed aggression (Fig. 3). This trend was inverted for siblings, which were tolerant of a similarly-sized counterpart, but were quickly aggressive in size-mismatched pairings. In other words, although large siblings were less aggressive overall, they had a shorter latency

393 different functions in different contexts. For example, when performed between size-mismatched 394 siblings, it may serve not to initiate an escalated fight but to ascertain by taste the first impression of relatedness. 
When considering latency to aggression, it may be important to take into account at which point tadpoles processed that they were not alone in the arena. The earliest occasion where this could have occurred is during the acclimation phase, during which tadpoles were separated by an opaque barrier which allowed water to pass through the entire testing arena (for an hour during acclimation, and

401 actively recorded for 10 minutes before the experiment). It is probable that chemical cues (if any) and 402 vibrations from tadpoles moving in the water circulated throughout the entire arena during this time. 403 Unexpectedly, half siblings were significantly less active than non-siblings and did not differ in 404 activity from siblings while dyads were separated (Fig 1, Panel A); relatedness-level differences in 405 activity disappeared once pairs could physically interact (Fig 1, Panel B). It should be noted that post406 acclimation behaviour was observed for only 10 minutes, and that tadpoles could have behaved 407 differently during the unobserved acclimation period (60 minutes). Nevertheless, higher activity levels 408 by non-siblings could indicate the presence of some kind of chemical cue whose meaning then shifted 409 with the presence of visual contact.

While we are unsure of the mechanisms $D$. tinctorius may be using to recognize each other, we believe 412 that tadpoles could potentially be using both olfactory and taste cues to discriminate kin, as shown in 413 salamanders (Pfennig et al., 1994) and Xenopus sp. (Dulcis et al., 2017). Fischer et al., (2020) recently 414 described the neural basis of conspecific aggression in D. tinctorius and found differences in brain 415 region activity based on the "winning" or "losing" status of paired tadpoles after fights; their findings 416 lay the groundwork for understanding the proximate mechanisms of aggression and provide the 417 backdrop to understanding its triggers in D. tinctorius tadpoles. Following the establishment of kin 418 discrimination in the species (this study), experiments investigating the possible mechanisms 419 underlying recognition are warranted. 


\section{Game theory model}

422 As intuitively expected, our model robustly predicts decreasing aggression levels with increasing 423 relatedness (Figure 4). Predictions about size-dependent behavior, however, turn out to be sensitive to 424 details. When aggression is assumed to be costlier for small tadpoles (bottom row of panels in Fig. 4), 425 larger tadpoles are always more aggressive, and are increasingly so towards larger size differences and 426 more distant relatedness. This formulation (1c) results in a pattern that is the most consistent with 427 experimental results (Fig 4). The model also reveals interesting theoretical possibilities which can be 428 rejected for D. tinctorius. For example, if (adult) reproductive success and aggressiveness costs were 429 independent of tadpole size, smaller tadpoles should compensate for their size disadvantage by being 430 more aggressive (top row of panels in Fig. 4). Moreover, if tadpole size strongly predicted adult 431 reproductive success, then above a certain size difference the smaller tadpole should let its larger 432 relative win without fighting (middle row of panels in Fig. 4).

\section{Conclusions}

435 In this study, we attempted to better understand the roles of physical attributes and relatedness in 436 predicting aggressive behavior under stable conditions. We found that large siblings were significantly 437 less aggressive than large non-siblings towards their smaller counterpart, demonstrating evidence for 438 kin discrimination in D. tinctorius. These findings are complicated by latency to aggression, which 439 showed different trends based on dyad relatedness, although the evolutionary explanation for this 440 pattern remains unclear. We contribute to the growing body of literature demonstrating aggressive 441 behavior (that may escalate to cannibalism) is independent of environmental stress, and conclude that 442 (1) size and relatedness interact in predicting aggressive behaviors, and (2) the ability to discriminate 443 kin does not guarantee kin bias. These findings set the stage for studies to consider cannibalistic 
444 behavior in more complex ways, and to better understand the value and purpose of kin discrimination 445 in cannibals.

\section{Acknowledgements}

448 We thank Teemu Tuomaala for taking care of tadpoles and the lab population; a big shout-out to 449 Aislyn Keyes for advice on coding the mating network which helped generate random pairs to keep 450 our frogs happy and healthy. Enormous thank you to Benjamin Leary for his amazing illustration 451 depicting tadpole aggression. This study was funded by the Academy of Finland (Academy Research 452 Fellowship No.21000042021 to BR).

\section{Statement of Authorship}

BR conceived the study, with input from CF and JV in the design of the assays; CF performed the study; LF developed the mathematical model; CF analyzed the data; and CF wrote the first draft with input from BR. All authors critically commented on previous drafts and approved the final version of the manuscript.

\section{BIBLIOGRAPHY}

Akaike, H. (1973). Maximum likelihood identification of Gaussian autoregressive moving average models. Biometrika 60, 255-265.

Van Allen, B.G., Dillemuth, F.P., Flick, A.J., Faldyn, M.J., Clark, D.R., Rudolf, V.H.W., and Elderd, B.D. (2017). Cannibalism and infectious disease: Friends or foes? Am. Nat. 190, 299-312.

Baras, E., Ndao, M., Maxi, M.Y.J., Jeandrain, D., Thomé, J.P., Vandewalle, P., and Mélard, C. (2000). Sibling cannibalism in dorada under experimental conditions. I. Ontogeny, dynamics, bioenergetics of cannibalism and prey size selectivity. J. Fish Biol. 57, 1001-1020.

Barkae, E.D., Golan, O., and Ovadia, O. (2014). Dangerous neighbors: Interactive effects of factors influencing cannibalism in pit-building antlion larvae. Behav. Ecol. 25, 1311-1319.

Baur, B. (1987). Effects of early feeding experience and age on the cannibalistic propensity of the land snail Arianta arbustorum. Can. J. Zool. 65, 3068-3070. 
Van den Beuken, T.P.G., Stockwell, L.W., and Smallegange, I.M. (2019). Et tu, brother? Kinship and increased nutrition lower the incidence of cannibalism in male bulb mites. Anim. Behav. $152,45-52$.

Bilde, T., and Lubin, Y. (2001). Kin recognition and cannibalism in a subsocial spider. J. Evol. Biol. 14, 959-966.

Bishop, A.M., Onoufriou, J., Moss, S., Pomeroy, P.P., and Twiss, S.D. (2016). Cannibalism by a male grey seal (halichoerus grypus) in the North Sea. Aquat. Mamm. 42, 137-143.

Boots, M. (2000). Kinship and cannibalism in the Indian meal moth, Plodia interpunctella: No evidence of kin discrimination. Evol. Ecol. Res. 2, 251-256.

Claessen, D., De Roos, A.M., and Persson, L. (2004). Population dynamic theory of size-dependent cannibalism. Proc. R. Soc. London B.

Cooper, W.E., Dimopoulos, I., and Pafilis, P. (2015). Sex, age, and population density affect aggressive behaviors in Island lizards promoting cannibalism. Ethology 121, 260-269.

Crump, M.L. (1990). Possible enhancement of growth in tadpoles through cannibalism. Copeia 560564.

Dobler, R., and Kölliker, M. (2011). Influence of weight asymmetry and kinship on siblicidal and cannibalistic behaviour in earwigs. Anim. Behav. 82, 667-672.

Dou, S., Seikai, T., and Tsukamoto, K. (2000). Cannibalism in Japanese flounder juveniles, Paralichthys olivaceus, reared under controlled conditions. Aquaculture 182, 149-159.

Dugas, M.B., Stynoski, J., and Strickler, S.A. (2016). Larval aggression is independent of food limitation in nurseries of a poison frog. Behav. Ecol. Sociobiol. 70, 1389-1395.

Dulcis, D., Lippi, G., Stark, C.J., Do, L.H., Berg, D.K., and Spitzer, N.C. (2017). Neurotransmitter Switching Regulated by miRNAs Controls Changes in Social Preference. Neuron 95, 13191333.e5.

Fischer, E.K., Alvarez, H., Lagerstrom, K.M., Petrillo, R., Ellis, G., and O'Connell, L.A. (2020). Neural correlates of winning and losing fights in poison frog tadpoles. Physiol. Behav. 2020.01.27.922286.

Fox, L.R. (1975). Cannibalism in Natural Populations. Annu. Rev. Ecol. Syst. 6, 87-106.

Gosner, K. (1960). A simplified table for staging anuran embryos and larvae with notes on $\mathrm{i}$ dentification. Herpetologica 16, 183-190.

Gray, H.M., Summers, K., and Ibáñez D., R. (2009). Kin discrimination in cannibalistic tadpoles of the Green Poison Frog, Dendrobates auratus (Anura, Dendrobatidae). Phyllomedusa 8, 4150 . 
Hartig, F. (2020). Package 'DHARMa .’

Ibáñez, C.M., and Keyl, F. (2010). Cannibalism in cephalopods. Rev. Fish Biol. Fish. 20, 123-136.

Jenkins, D.W., and Carpenter, S.J. (1946). Ecology of the Tree Hole Breeding Mosquitoes of Nearctic North America. Ecol. Monogr. 16, 31-47.

Magnusson, A., Hans, S., Nielsen, A., Berg, C., Kristensen, K., Marchler, M., van Bentham, K., Bolker, B., and Brooks, M. (2020). Package ' glmmTMB .'

Margalida, A., Bertran, J., Boudet, J., and Heredia, R. (2004). Hatching asynchrony, sibling agression and cannibalism in the Bearded Vulture Gypaetus barbatus. Ibis (Lond. 1859). 146, 386-393.

Mayntz, D., and Toft, S. (2006). Nutritional value of cannibalism and the role of starvation and nutrient imbalance for cannibalistic tendencies in a generalist predator. J. Anim. Ecol. 75, 288-297.

Merritt Hawkes, O.A. (1920). Life-history of the lady-bird beetle. Proc. Zool. Soc. London 475-490.

Mock, D.W., Lamey, T.C., and Ploger, B.J. (1987). Proximate and Ultimate Roles of Food Amount in Regulating Egret Sibling Aggression. Ecology 68, 1760-1772.

Moksnes, P.O. (2004). Self-regulating mechanisms in cannibalistic populations of juvenile shore crabs Carcinus maenas. Ecology 85, 1343-1354.

Pfennig, D.W. (1999). Cannibalistic tadpoles that pose the greatest threat to kin are most likely to discriminate kin. Proc. R. Soc. B Biol. Sci.

Pfennig, D.W., and Frankino, W.A. (1997). Kin-Mediated Morphogenesis in Facultatively Cannibalistic Tadpoles. Evolution (N. Y). 51, 1993.

Pfennig, D.W., Loeb, M.L.G., and Collins, J.P. (1991). Pathogens as a factor limiting the spread of cannibalism in tiger salamanders. Oecologia $88,161-166$.

Pfennig, D.W., Sherman, P.W., and Collins, J.P. (1994). Kin recognition and cannibalism in polyphenic salamanders. Behav. Ecol. 5, 225-232.

Polis, G.A. (1981). The Evolution and Dynamics of Intraspecific Predation. Annu. Rev. Ecol. Syst. $12,225-251$.

Richardson, M.L., Mitchell, R.F., Reagel, P.F., and Hanks, L.M. (2010). Causes and Consequences of Cannibalism in Noncarnivorous Insects. Annu. Rev. Entomol. 55, 39-53.

Roberts, J.A., Taylor, P.W., and Uetz, G.W. (2003). Kinship and food availability influence cannibalism tendency in early-instar wolf spiders (Araneae: Lycosidae). Behav. Ecol. Sociobiol. 54, 416-422.

Rojas, B. (2014). Strange parental decisions: Fathers of the dyeing poison frog deposit their tadpoles 
in pools occupied by large cannibals. Behav. Ecol. Sociobiol. 68, 551-559.

Rojas, B. (2015). Mind the gap : treefalls as drivers of parental trade-offs. Ecol. Evol. 5, 4028-4036.

Rojas, B., and Pašukonis, A. (2019). From Habitat Use to Social Behavior : Natural History of a Voiceless Poison Frog, Dendrobates tinctorius. PeerJ 1-30.

Schulte, L.M., and Mayer, M. (2017). Poison frog tadpoles seek parental transportation to escape their cannibalistic siblings. J. Zool. 303, 83-89.

Schulte, L.M., Yeager, J., Schulte, R., Veith, M., Werner, P., Beck, L.A., and Lötters, S. (2011). The smell of success: Choice of larval rearing sites by means of chemical cues in a Peruvian poison frog. Anim. Behav. 81, 1147-1154.

547

548

Stamps, J.A., and Krishnan, V. V. (1994). Territory acquisition in lizards: I. First encounters. Anim. Behav. 47, 1375-1385.

Summers, K., and Symula, R. (2001). Cannibalism and kin discrimination in tadpoles of the amazonian poison frog, Dendrobates ventrimaculatus, in the field. Herpetol. J. 11, 17-21.

Takahata, Y. (1985). Adult male chimpanzees kill and eat a male newborn infant: Newly observed intragroup infanticide and cannibalism in mahale national park, Tanzania. Folia Primatol. 44, 161-170.

Team, R.C. (2019). R: A language and environment for statistical computing. R Found. Stat. Comput. Vienna, Austria.

Tershy, B.R., Breese, D., and Croll, D.A. (2000). Insurance eggs versus additional eggs: Do brown boobies practice obligate siblicide. Auk 117, 817-820.

Therneau, T.M. (2020). Package ' coxme .'

Wang, X.G., and Daane, K.M. (2014). Cannibalism of parasitoid-attacked conspecifics in a noncarnivorous caterpillar. Entomol. Exp. Appl. 151, 112-121.

Wickham, H., François, R., Henry, L., and Müller, K. (2018). Package “dplyr.”

Wickham, H., Averick, M., Bryan, J., Chang, W., McGowan, L., François, R., Grolemund, G., Hayes, A., Henry, L., Hester, J., et al. (2019). Welcome to the Tidyverse. J. Open Source Softw. 4, 1686. 